\title{
Fuzzy Logic Model for Initial Project Screening with Consideration of Decision Position
}

\author{
Li-Chung Chao ${ }^{\mathrm{a}, *}$ \\ ${ }^{a}$ National Kaohsiung University of Science and Technology,1 University Road, Yanchao Kaohsiung 824, Taiwan ROC
}

\begin{abstract}
In planning for a construction project, the owner often has several alternatives regarding the site or the building that are available for selection. Evaluation of the project alternatives and then ranking them in preference so as to select the overall best one for implementation is the key issue that influences project success. This paper proposes a model for initial screening of project alternatives with consideration of the owner's decision position that is reflected by the priority differences between the decision factors. The model uses the fuzzy inference system to perform mapping from the estimates of the factors or the inputs for an alternative to its score or the output. In order to illustrate the model, hypothetical alternative sites of a housing project for a developer firm are assumed and an example fuzzy inference system is built to simulate evaluation and ranking of them. First, three variables, i.e. project size, project conditions, and unit development cost, are used as the input variables that determine the desirability of a site in initial project screening. Next, the linguistics values of the input and output variables each are defined with a set of membership functions. Then, the fuzzy rules that represent the owner's decision position in a possible scenario are set up. For given inputs, the output is produced by mathematical operations on the rules. The assessments and rankings obtained are found to be consistent with the inputs for the sites and the decision position, showing that the model can capture the effect of nonlinear input-output relations and is potentially useful for initial project screening.
\end{abstract}

(C) 2018 The Authors. Published by Diamond Congress Ltd., Budapest University of Technology and Economics Peer-review under responsibility of the scientific committee of the Creative Construction Conference 2018.

Keywords: decision position; evaluation; fuzzy logic; housing development.

\section{Introduction}

In planning for a construction project, whether in the public or private sector, the owner often has several alternatives that are available for selection; for example, a few sites available for development and at a site different types of building available for erection. Performing analysis of the alternatives and ranking them so as to select the overall best one for implementation is the key issue that influences success of the project. There have been many cases of project failures due to improper project selection, resulting in wastes of valuable resources. The existing methods for project assessment, such as the net present worth method, the utility theory method, and the analytic hierarchy process method, each have some disadvantages.

The traditional net present worth (NPW) method is based on the estimated cash flows of incomes and expenses for the alternatives, but it is difficult to include intangible benefits and risk factors and so the result may not represent their overall values. The utility theory method, which is multi-attribute decision analysis (MADA), can include quantitative and qualitative elements as evaluation criteria and produces a total utility score for each alternative [1,2]. However, it has difficulty in determining criteria weights and utility functions. The analytic hierarchy/network process (AHP/ANP) method structures the evaluation as a hierarchy/network to contain all criteria and uses paired comparisons to generate criteria weights and then aggregates the alternatives' overall scores [3,4]. However, the

Corresponding author: Author email: chaolc@nkfust.edu.tw 
AHP/ANP method has no explicit functions in producing ratings and so lacks transparency. To redress the above shortcomings of the existing methods is the motivation for the present research.

The objective of this research is to develop an improved model for assessing and ranking alternatives in initial project screening. For the purpose of initial project screening before a final selection is made, the model should be able to differentiate the more favorable ones from the less favorable ones. Therefore, the model needs to include only the main factors, both quantitative and qualitative. Moreover, the model needs to address the decision position of the project owner that is reflected by the priority differences between the factors.

\section{Research method}

It is proposed to use fuzzy logic (also called fuzzy inference) for mapping of the output (score) for an alternative from the inputs (factors) for it, because project assessment involves great complexities and fuzzy logic can deal with complex nonlinear input-output relations. Fuzzy logic has found applications in many areas of construction [5,6,7]. It is based on the concept of fuzzy sets or membership functions for describing the values of linguistic variables. Fuzzy rules in the form of IF preconditions THEN consequences are employed to emulate the linguistic way that humans judge in dealing with different circumstances. An inference is achieved by mathematical operations on the rules to determine the output for given inputs. Steps of the inference process include fuzzifying crisp inputs, calculating rules' firing strengths, weighing consequences, aggregating weighed consequences, and defuzzifying the result into crisp outputs. Through the collective effect of the fuzzy rules on the output for given inputs, fuzzy logic can accommodate imprecise data and achieve robustness. The strength of the fuzzy logic is to produce consistent results, while keeping to tractability [8].

Selection of alternative sites by a developer for housing development is used herein as an example to illustrate the proposed model for initial screening of project alternatives. From a previous study [4], three important factors that influence a site's overall strength are identified as the criteria for evaluating alternative sites. The first factor is project size, which refers to the gross floor area as planned for a site according to its acreage and land use regulations. The second factor is project conditions, which are defined by three sub-factors. The first sub-factor is surroundings conditions, which refer to a site's external environments including completeness of transport systems, access to public transports, availability of public facilities such as schools, markets, and services, and distance to unfavorable places such as cemetery and landfills. The second sub-factor is plot use and construction plan, which refer to a site's suitability for housing development and construction concerning the plot shape, terrain, and ground conditions. The third sub-factor is local demand and competitiveness, which refer to the expected demand and competition for sales of new housing in the local area. The last and third factor is total development cost, which refers to all expenses needed in the project as planned for a site and comprises purchase of the site, planning and design, construction, management and marketing.

In the present study, hypothetical alternative sites for a housing development project in Kaohsiung, Taiwan are assumed for a developer and used for illustrating the proposed fuzzy logic model by simulation of assessment and ranking of them in priority. The framework of the model for initial screening of alternative sites for a housing project is presented in the following section.

\section{Description of model}

For an overall assessment of alternative sites for housing development, the three factors mentioned above, i.e. project size, project conditions, and unit development cost, are used as the input variables for building the proposed evaluation model. Project size is defined as the planned gross floor $\left(\mathrm{m}^{2}\right)$ for a site. Project conditions are a qualitative factor and assessed on a scale of 0-100 measuring the degree of favorableness of a site by considering surroundings conditions, plot use and construction plan, and local demand and competitiveness together. Unit development cost $\left(\mathrm{NT} \$ / \mathrm{m}^{2}, 1 \mathrm{NT} \$ 0.03 \mathrm{US} \$\right.$ ) is defined as the estimated total development cost divided by the planned gross floor area for a site. The output variable of the model is the score assessed for a site on a scale of 0-1. See Fig. 1 for the input and output variables of the model.

In fact, there are two inferences involved in the model. The first inference is to assess the project conditions of a site based on the site's ratings of surroundings conditions, plot use and construction plan, and local demand and competitiveness. The second inference is to produce the overall assessment of a site based on the site's project size, project conditions assessment, and unit development cost. However, for the sake of simplicity, only the second 
inference is presented in the example herein, while the result of the first inference for a site is assumed as a $0-100$ rating.

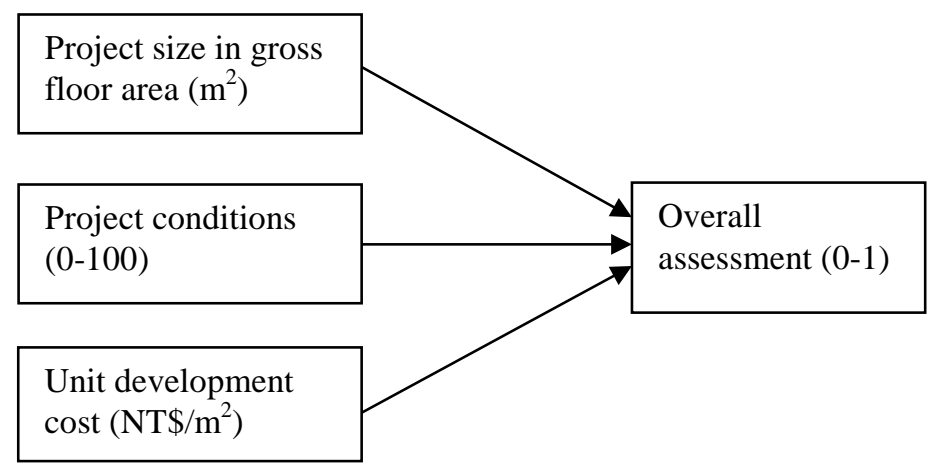

Fig. 1. Inputs and output of model for evaluating alternative sites.

The membership functions, fuzzy rules, and mathematical operations used in the inference for overall assessment are explained below. The linguistic values or levels of each input and output variable in the fuzzy rules are defined with a set of membership functions separately. It is proposed to use the Gaussian membership function to describe the levels of each input variable, which are the preconditions of the rules. The range of value of an input variable or the domain of the membership functions is set based on the possible project cases for a developer. The parameters of a membership function are set so that the adjacent curves intersect at membership of 0.5 and the overlapping can cause the consequences of quite a few rules to impact the output collectively. The Gaussian membership function is defined in (1) below:

$$
m=f(x, \sigma, c)=e^{\frac{-(x-c)^{2}}{2 \sigma^{2}}}
$$

where $x=$ value of an element in the domain of function $f$; $m=$ value of membership function $f$ at $x$ in $f$; $c$ position of the center of the peak; $\sigma=$ standard deviation.

Assuming that project size's range is 1,000 100,000 $\mathrm{m}^{2}$, the three membership functions in Figure 2(a) are used to define the three linguistics values, where $\sigma=21000, c=1000$ (small), 50500 (medium), or 100000 (large). As project conditions are assessed on a scale of $0-100$, the three membership functions in Figure 2(b) are used for the variable, where $\sigma=21.25, c=0$ (poor), 50 (average), or 100 (excellent).

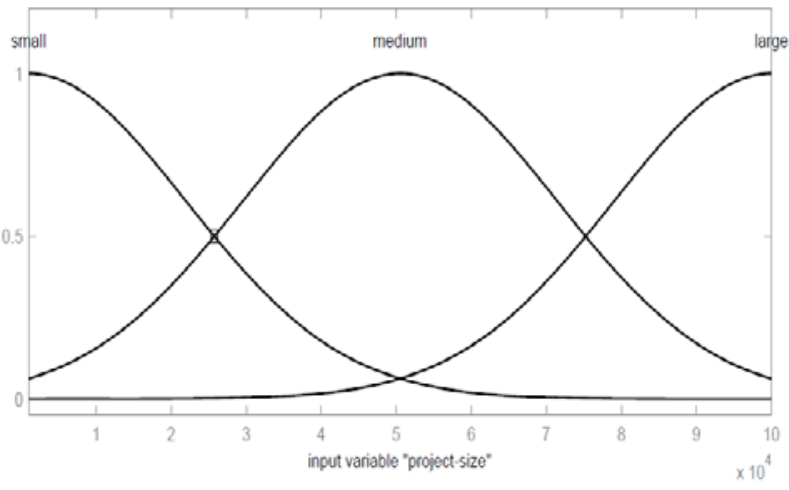

(a)

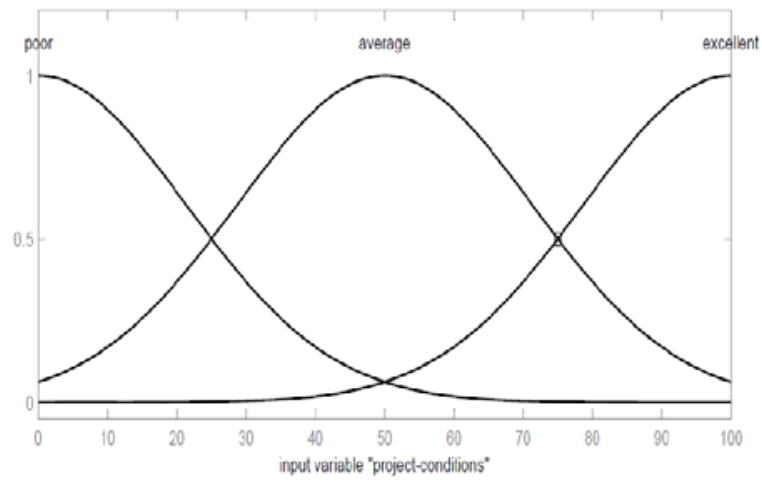

(b)

Fig. 2. Membership functions for fuzzy variables (a) “project size” (b) “project conditions”.

Assuming that unit development cost's range is $30,000 \sim 300,000 \mathrm{NT} \$ / \mathrm{m}^{2}$, the three membership functions in Figure 3(a) are used for the variable, where $\sigma=57500, c=30000$ (low), 165000 (medium), or 300000 (high). It is proposed to use the triangular membership function to describe the levels or ranks of the output variable "overall 
assessment”, which are the consequences of the rules. As the score of a site is assessed on a scale of $0-1$, the three membership functions in Figure 3(b) are used for the variable, where base width $=0.5$, center $=0$ (E), 0.25 (D), 0.5 (C), $0.75(\mathrm{~B})$ or $1(\mathrm{~A})$.

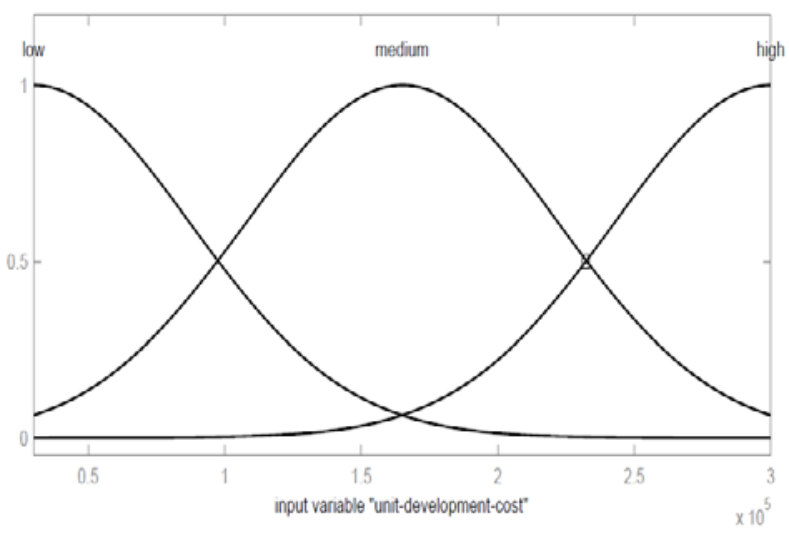

(a)

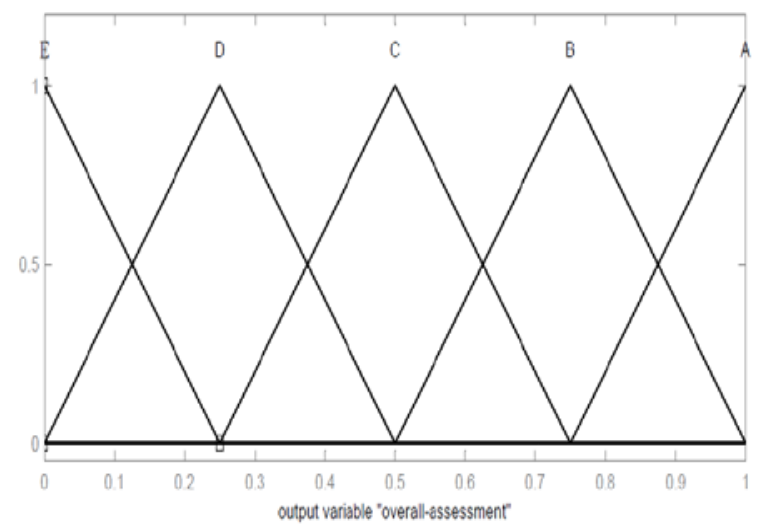

(b)

Fig. 3. Membership functions for fuzzy variables (a) “unit development cost” (b) “overall assessment”.

Addressing the developer's decision position in a possible scenario, the set of fuzzy rules that reflect the decision position by the priority differences between the input variables are set up for producing a score for given inputs of the project size, project conditions assessment, and unit development cost for an alternative site. The preconditions of each rule consist of the linguistic values of the input variables, i.e. a level of project size (small, medium, or large), a level of project conditions assessment (poor, average, or excellent), and a level of unit development cost (low, medium, or high). The connection between the preconditions can be the "AND" operator or the "OR" operator, while some preconditions in a rule may be absent. The consequence part of each rule is a rank from " $A$ " to " $E$ " assigned for the preconditions.

The reality of housing development is that a site with better project conditions has a greater potential for sales but also requires a higher development cost because of a higher land price and causes more concern. Although a site with poorer project conditions has a less potential for sales, it requires a lower development cost because of a lower land price and causes less concern. Whether the priority is project conditions or unit development cost or the two factors are equally important depends on decision position, which is reflected by a set of fuzzy rules. Besides, the optimal project size depends also on decision position; it is not necessary that the larger the better because of the concern about the higher financial risk due to greater possibility of more unsold units for a greater project size. Assume a scenario of the developer pursuing higher sales brought by better project conditions of a site but also having an aversion to greater concern caused by higher unit development cost, meanwhile considering medium project size best, followed by small project size and large project size. Then, a set of fuzzy rules suitable for the decision position are set up as shown in Table 1.

Table 1. Example fuzzy rules for evaluating alternative sites

\begin{tabular}{llll}
\hline Project size & Project conditions & Unit development cost & Rank \\
\hline Medium & Excellent & Low & A \\
Medium & Average & Medium & B \\
Medium & Poor & High & C \\
Small & Excellent & Low & B \\
Small & Average & Medium & C \\
Small & Poor & High & D \\
Large & Excellent & Low & C \\
Large & Average & Medium & D \\
Large & Poor & High & E \\
\hline
\end{tabular}


In Table 1, the first rule reads "for a alternative site being assessed, if the project size is medium, and the project conditions are excellent or the unit development cost is low, then its rank is A." It has the effect of two rules: "if the project size is medium and the project conditions are excellent, then the rank is A" and "if the project size is medium and the unit cost development cost is low, then the rank is A.” Therefore, the rank A is assigned to a site that meets the preconditions of any one of the two rules. The remaining eight rules have similar effects. These rules indicate that the assigned rank generally turns worse following the order of medium, small, large project size and within each level of project size the rank turns worse with poorer project conditions or higher unit development cost. For this decision position, the site with the highest score obtained from the fuzzy inference is likely the one with all inputs close to the middles, representing a compromise.

A set of fuzzy rules, the membership functions for the rules, as well as the mathematical operations used in fuzzy inference comprise a fuzzy inference system (FIS). In the illustrative example FIS, the process of transforming the inputs for an alternative site to the output (score) for it is explained in the steps below.

1. Let the memberships of $k$ inputs in the preconditions of rule $i$ be $m_{1}, m_{2}, \ldots, m_{k}$, respectively, then either (2) or (3) below is used to calculate the firing strength of rule $i\left(F_{i}\right)$.

For the "AND" connection between the preconditions of rule $i$,

$$
F_{i}=\min \left(m_{1}, m_{2}, \ldots, m_{k}\right)
$$

where operation min stands for minimization.

For the "OR" connection between the preconditions of rule $i$,

$$
F_{i}=\max \left(m_{1}, m_{2}, \ldots, m_{k}\right)
$$

where operation max stands for maximization.

2. The obtained firing strength $F_{i}$ from step 1 is used to weigh the consequence membership function of rule $i, m_{r i}(x)$, which is the rank assigned. The step is called implication and the result of it is:

$$
s_{i}(x)=\min \left(F_{i}, m_{r i}(x)\right)
$$

3. By aggregating the weighed consequences of all $n$ rules, the obtained overall results is:

$$
S(x)=\max \left(s_{1}(x), s_{2}(x), \ldots, s_{n}(x)\right)
$$

4. The final step is to defuzzify the result of step 3 by calculating the $x$ value of the centroid or the center of the area defined by $S(x)$, which is very likely a polygon, to produce the score of the site, and the score is a value between 0 and 1.

For building the fuzzy inference system above, MATLAB's Fuzzy Logic Toolbox (MATLAB, 2014) can be used because its graphical user interfaces facilitates formulation and revision in model development. Sets of fuzzy rules for overall assessment of alternative sites in other scenarios and those for project conditions assessment from its subfactors can be set-up similarly according to corresponding decision positions. When the model is put to use, the estimates of the input variables for each alternative site are made and entered into respective FIS to produce the overall assessments of the alternative sites as the outputs for a decision position.

\section{Example site assessment and results}

It is assumed that a developer with a modest financial capacity in planning a housing development project has five hypothetical alternative sites, A, B, C, D, and E available for selection. The proposed fuzzy logic model is used for simulation of assessment and ranking of the sites in initial project screening. The values of the input variables shown in Table 2, i.e. project size, project conditions, and unit developments cost for each site, are entered into the above FIS to produce the scores of the sites. The results are also shown in Table 2; site $\mathrm{C}$ with the highest score ranks first, followed by site A and site B, while site E and site D rank lower. The rankings obtained are consistent with the site' strengths and weaknesses from the viewpoint of the decision position or the priority differences between the factors as reflected by the fuzzy rules in Table 1; project size is the most important factor, while project conditions and unit development cost are equally and less important factors. In comparison with other MADA methods, the fuzzy logic model has an important advantage for the example: it would be difficult to use utility functions in the form of a 
smooth curve to achieve the effect of order of preference for project size: medium first, small next, and large last, while using AHP/ANP still have the problem of lacking transparency in the ratings entered.

Table 2. Project size, project conditions, unit development cost, and score of the alternative sites in the illustrative example

\begin{tabular}{lllll}
\hline Alternative site & Project size $\left(\mathrm{m}^{2}\right)$ & Project conditions $(0-100)$ & Unit development cost $\left(\mathrm{NT} \$ / \mathrm{m}^{2}\right)$ & Score $(0-1)$ \\
\hline Site A & 60,000 & 70 & 160,000 & 0.649 \\
Site B & 30,000 & 85 & 200,000 & 0.618 \\
Site C & 45,000 & 60 & 120,000 & 0.708 \\
Site D & 75,000 & 45 & 80,000 & 0.534 \\
Site E & 70,000 & 55 & 85,000 & 0.589 \\
\hline
\end{tabular}

\section{Conclusions}

Initial project screening is important for construction owners since it influences the final outcome of a project. A fuzzy logic model is proposed in the paper for assessment and ranking of alternative sites for a housing development project. The model comprises membership functions for the input and output variables, fuzzy rules incorporating decision position by reflecting the priority differences between the input variables, and mathematical operations for processing inputs and producing the output. The model is illustrated with an example, in which project size, project conditions, and unit development cost are used as input variables and five sites are assessed. The results obtained are consistent with the sites' strengths and weaknesses from the viewpoint of a possible decision position. As compared to other methods, the model can better capture non-linear effects of the decision position. It is emphasized that the result from the model can only be considered as suggestion of initial screening, and so more in-depth study of those accepted in the screening should be done in order to determine the final selection with other decision factors not included in the model.

\section{References}

[1] M.W. Lifson, J.E.F. Shaifer, Decision and Risk Analysis for Construction Management. JohnWiley and Sons, 1982.

[2] L.-C. Chao, M.J. Skibniewski, Decision analysis for new construction technology implementation, Civil Engineering Systems. 12 (1995) 6782.

[3] M.J. Skibniewski, L.-C. Chao, Evaluation of advanced construction technology with AHP method, Journal of Construction Engineering and Management. 118(3) (1992) 577-593.

[4] L.-C. Chao, Evaluation of alternative construction sites with analytic network process method, Proceedings of The 12th International Symposium on the Analytic Hierarchy Process. (2013).

[5] L.-C. Chao, M.J. Skibniewski, Fuzzy logic for evaluating alternative construction technology, Journal of Construction Engineering and Management. 124(4) (1998) 297-304.

[6] J.H.M. Tah, V. Carr, A proposal for construction project risk assessment using fuzzy logic, Construction Management and Economics. 18 (2000) 491-500.

[7] X.-H. Jin, H. Doloi, Modeling risk allocation in privately financed infrastructure projects using fuzzy logic, Computer-Aided Civil and Infrastructure Engineering. 24(7) (2009) 509-524.

[8] L.A. Zadeh, Foreword, Fuzzy Logic Toolbox for Use with MATLAB, User's Guide Version 2, The Math Works, Inc., 1995.

[9] MATLAB, Fuzzy Logic Toolbox for Use with MATLAB R2014a, User’s Guide, The Math Works, Inc., 2014. 